\title{
February 2014 Pulmonary Case of the Month: Faster Is Not Always Better
}

\author{
Lewis Wesselius MD \\ Department of Pulmonary Medicine \\ Mayo Clinic Arizona \\ Scottsdale, AZ
}

\section{History of Present IIIness}

A 56 year old woman with a history of rheumatoid arthritis (RA) for 26 years was seen as an outpatient. She has a recent history of increased cough, sputum and dyspnea.

\section{$\mathrm{PMH}, \mathrm{FH}, \mathrm{SH}$}

She was originally from India but had lived in Singapore from 2011 to June 2013 before moving to Phoenix. In 2009, she was diagnosed with Mycobacterium avium-intracellulare (MAI) on bronchoscopy and started on azithromycin, ethambutol, and rifabutin. She was unable to tolerate rifabutin but was continued on ethambutol and azithromycin. She had been on etanercept for her RA which was held after the diagnosis of MAl. She had negative sputum cultures for MAI in September 2012 and her ethambutol and azithromycin were stopped. In May 2013 she had increased symptoms and bronchoscopy demonstrated Pseudomonas and nontuberculous mycobacterium (NTM). She was treated with cefipime/ciprofloxacin for 6 weeks prior to moving to Phoenix.

She does not smoke or drink. Her FH is noncontributory.

\section{Medications}

- Celecoxib $200 \mathrm{mg}$ bid

- Gabapentin 600 bid

- Methotrexate $15 \mathrm{mg}$ weekly

- Prednisone $5 \mathrm{mg}$ daily

- Tramadol 50 mg every 4 hours prn

\section{Physical Examination}

Afebrile. SpO2 96\% on room air.

Chest: scattered crackles in both lungs, no wheezes.

There were joint deformities typical of chronic RA, but otherwise the remainder of the physical exam was unremarkable.

\section{Radiology}

She brings a CT scan from 2009 (Figure 1). 


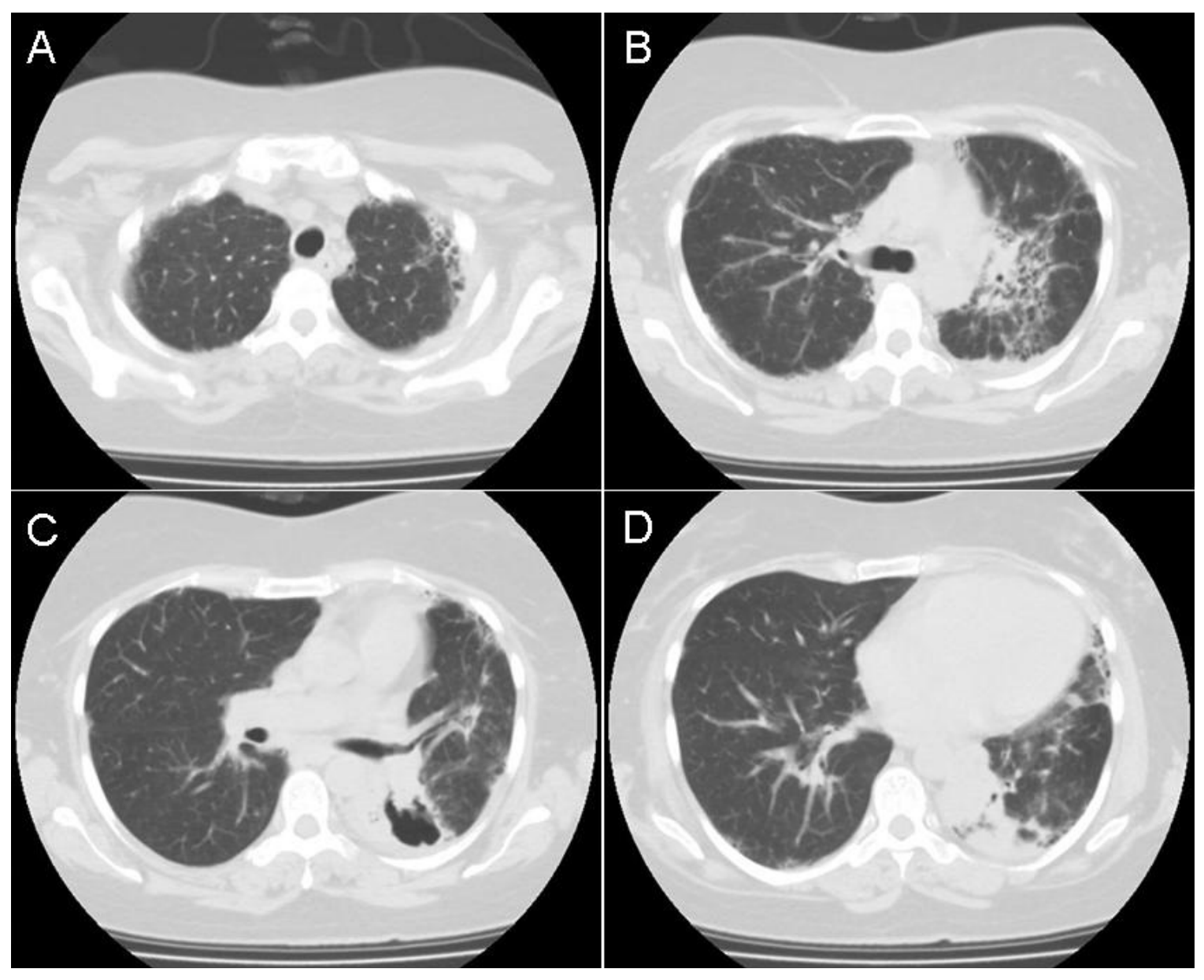

Figure 1. Representative static axial lung images from a thoracic CT scan performed in 2009.

What should be done next?

1. Repeat the CT scan

2. Restart ethambutol and azithromycin

3. Sputum culture

4. $1+3$

5. All of the above 


\section{Correct!}

\section{4. $1+3$}

The cause of her increasing symptoms is not clear from her history and physical examination and her old CT scan. For this reason a repeat of her CT scan and sputum culture are indicated. Her repeat CT scan is shown in Figure 2.

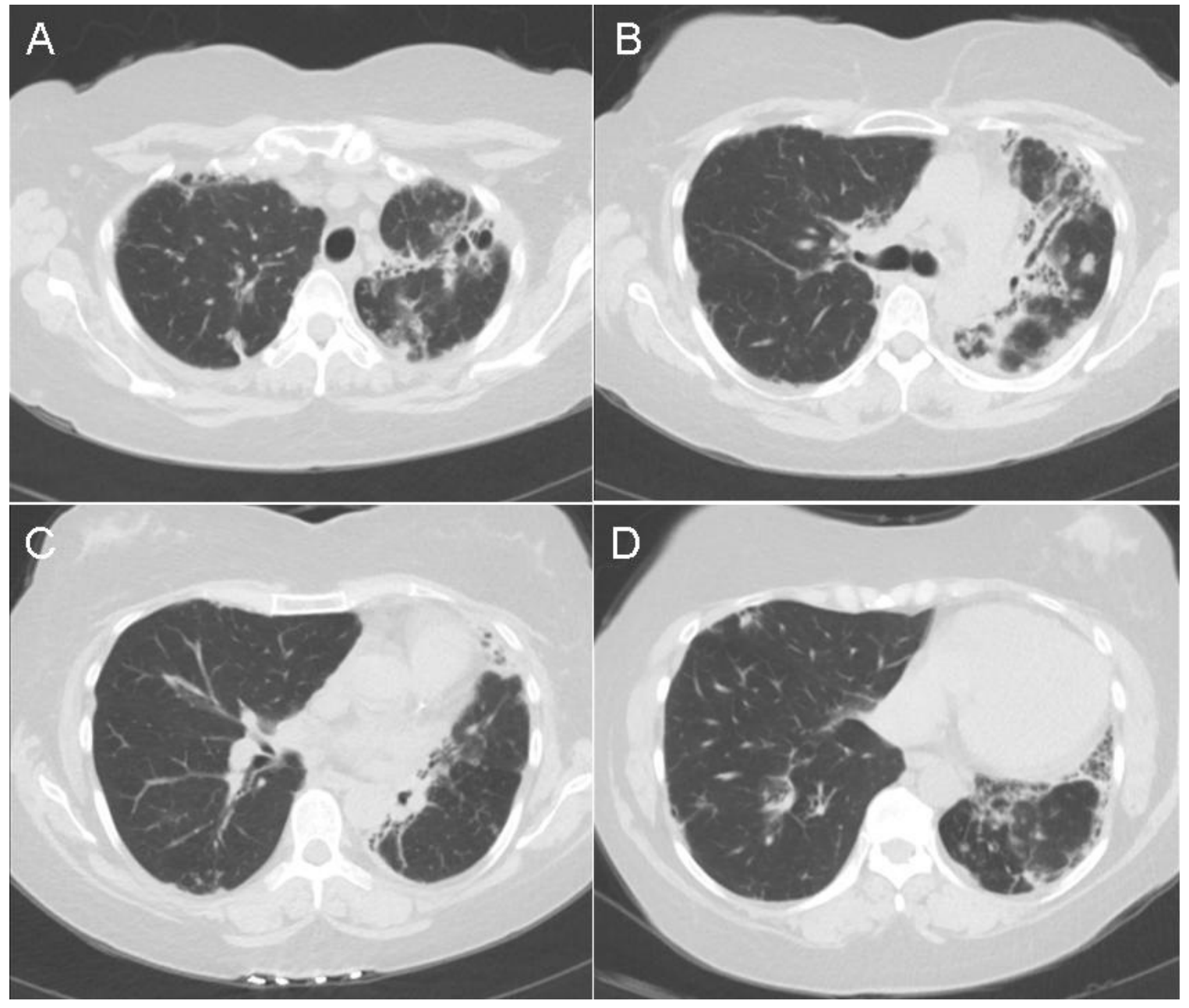

Figure 2. Representative static axial lung images from a repeat thoracic CT scan.

Which of the following disorder(s) associated with rheumatoid arthritis (RA) is seen in the patient's thoracic CT scan?

1. Bronchiectasis

2. Obliterative bronchiolitis

3. Pleural effusion

4. Pulmonary hypertension

5. Rheumatoid nodules 


\section{Correct! \\ 1. Bronchiectasis}

All the listed disorders are associated with rheumatoid arthritis (1). There is no evidence of pleural effusion, rheumatoid nodules or pulmonary hypertension (dilated pulmonary arteries, enlarged right ventricle) on the CT scan. Rheumatoid lung fibrosis is more common in men than in women. Most commonly lung fibrosis in rheumatoid arthritis presents as usual interstitial pneumonia or nonspecific interstitial pneumonia. There is a recognized association between rheumatoid disease and obliterative bronchiolitis (constrictive bronchiolitis) in which bronchioles are destroyed and replaced by scar tissue. The characteristic CT findings are mosaic perfusion with expiratory air trapping.

The association between rheumatoid arthritis and bronchiectasis was first reported in 1955, but was considered rare until the 1990s (2). The prevalence of bronchiectasis in RA reported to range from $5.6 \%$ to $30 \%$ in prospective blinded studies with HRCT. RA is diagnosed in $2.7-5.2 \%$ of patients with bronchiectasis being seen in pulmonary clinic. The mechanism(s) underlying the association of these conditions remain unclear, but recent studies indicate an association between bronchiectasis developing in RA patients and the presence of CFTR mutations. Bronchiectasis has been found to precede RA by 16-29 years. In addition, the use of use of nonbiological and biological disease-modifying antirheumatic drugs likely increase the rate of lower respiratory tract infection and colonization of the airways increasing the incidence of bronchiectasis.

Our patient's CT scan shows bronchiectasis with cylindrical bronchial dilatation and bronchial wall thickening. Her sputum culture grew Pseudomonas aeruginosa and Mycobacterium abscessus.

Which of the following is (are) true regarding nontuberculous (NTB) infection in bronchiectasis?

1. Mycobacterium abscessus and Mycobacterium kansasii are increasing in prevalence

2. Mycobacterium abscessus is usually easily controlled with macrolide antibioitics

3. Mycobacterium avium-intracellulare is the most common NTB isolated

4. $1+3$

5. All of the above 


\section{Correct!}

\section{4. $1+3$}

Mycobacterium avium-intracellulare is most common NTM isolated from bronchiectasis patients (2). However, Mycobacterium abscessus and Mycobacterium kansasii are increasing in prevalence in recent studies. The largest study described 154 patients with rapidly growing mycobacterial infection, and $119(82 \%)$ of 146 respiratory isolates identified were Mycobacterium abscessus (3). Mycobacterium abscessus is inherently multidrug resistant and, therefore, challenging to treat. There have been no controlled studies conducted for the treatment of rapidly growing mycobacteria infection. Current treatment recommendations include multidrug therapy with combinations of intravenous and oral antibiotics and/or surgery.

Our patient was Mycobacterium abscessus was only sensitive to amikacin. She was treated with IV tigecycline and inhaled amikacin. She has modest clinical improvement.

\section{References}

1. Lynch DA. Lung disease related to collagen vascular disease. J Thorac Imaging. 2009;24(4):299-309. [CrossRef] [PubMed]

2. Wilczynska MM, Condliffe AM, McKeon DJ. Coexistence of bronchiectasis and rheumatoid arthritis: revisited. Respir Care. 2013;58(4):694-701. [CrossRef] [PubMed]

3. Jarand J, Levin A, Zhang L, Huitt G, Mitchell JD, Daley CL. Clinical and microbiologic outcomes in patients receiving treatment for Mycobacterium abscessus pulmonary disease. Clin Infect Dis. 2011;52(5):565-71. [CrossRef] [PubMed] 